\title{
An investigation on RF CMOS stability related to bias and scaling
}

\author{
Jiong-Guang Su ${ }^{\text {a }}$, Shyh-Chyi Wong ${ }^{\mathrm{b}}$, Chun-Yen Chang ${ }^{\mathrm{a}, *}$ \\ a Department of Electronics Engineering, Institute of Electronics, National Chiao Tung University, 1001, Ta Hsueh Rd., Hsinchu 300, \\ Taiwan, ROC \\ ${ }^{\mathrm{b}}$ Research Development, Taiwan Semiconductor Manufacturing Co., 9, Creation Rd. 1, Science-Based Industrial Park, Hsinchu 300, \\ Taiwan, $R O C$
}

Received 9 April 2001; accepted 19 October 2001

\begin{abstract}
In recent times, CMOS devices have played an increasing important role in the area of RF-ICs to implement part of the high-frequency circuits. As a result, the high-frequency characteristics of CMOS devices, including stability, should be investigated in-depth. In the design of RF amplifier, the consideration to avoid oscillation in the circuits is a must. Basically, the instability involved in designing RF circuits can be reduced if the transistor is stable in the interesting frequency region. In this study, the stability factor of CMOS device is discussed. First, the stability factor (i.e. $k$-factor) is derived based on the microwave small-signal model of MOSFET. Therefore, small-signal parameters that affect stability can be carefully examined. In addition, the effects of biasing and dimensions on $k$-factor are also discussed. Since various operating point (or biasing) can cause device to have different effects on high frequency, the correlation between biasing and stability is discussed in this study. Due to the fact that CMOS device has continuously shrunk in size, a detailed discussion of the effect of MOSFETs dimension on stability is included. It is found from our experimental results that the smaller the gate length, the greater is the frequency range for CMOS device to become potentially unstable. (c) 2002 Elsevier Science Ltd. All rights reserved.
\end{abstract}

Keywords: RF-ICs; CMOS; Stability factor; RF amplifier

\section{Introduction}

In recent years CMOS technologies have become viable and continued its expansion in the area of RF circuit implementations as a result of the advancement of aggressive scaling, progressiveness on high-speed performance and reduction in cost. In addition, system-ona-chip (SOC) can be realized by integrating memories, processor cores, logic, and analog cores into the same silicon chip after successfully implementing front-end circuits of RF CMOS [1]. Hence, the methodology of designing the MOSFET device for logic and analog circuits in conjunction with RF applications has to be

\footnotetext{
${ }^{*}$ Corresponding author.

E-mail address: cyc@cc.nctu.edu.tw (C.-Y. Chang).
}

investigated. In general, the following considerations should be considered when designing a RF active circuit and they are dc bias, noise, bandwidth, power gain, harmonic distortion, stability, and impedance matching [2,3]. Most of these circuit performances are strongly dependent on and restricted by transistor's RF properties. Hence, the RF characteristics of CMOS are the main focus to be investigated in designing device and circuit.

Recently, electrical parameters such as unity current gain cut-off frequency $\left(F_{\mathrm{t}}\right)$, maximum oscillation frequency $\left(F_{\max }\right)$, maximum stable power gain (MSG), maximum available power gain (MAG), flicker noise, high-frequency noise figure, linearity and related dc power consumption have been discussed in pursuit of RF characteristics from CMOS devices [4-8]. However, there is still a lack of the decisive study with respect to the parameter of stability in these literatures. 
Basically, the stability is one of the most important factors in designing RF amplifiers. An amplifier should be fabricated to be absolutely stable within a specific frequency range. Transistors with unconditionally stable will make the work of RF amplifier design easily. In fact, transistors with potentially stable also can be used for RF amplifier design. Additional matching network can be used to take the matching point far from the unstable region. However, difficulty of circuit design will be raised, and the RF performance such as power gain and noise level will be degraded. Besides, for working in the stable region, passive components, which can be used in matching network, will be limited. Requirements on precise and scalable passive components will be severe, and this eventually hard to fabricate in ICs. Therefore, the device's stability is extremely important for implementing RF amplifiers successfully from technology's perspective. Unfortunately, MOSFET exhibits poor stability for RF active circuit design and this is especially serious while extend the technology to submicron devices. The obviously observation is the evaluation of maximum oscillation frequency from MSG and MAG. $F_{\max }$ is difficult to extracted from MSG and MAG because that $k>1$ occurs at extremely high frequency.

In 1976, Woods discussed the stable criteria required to be unconditionally necessary and sufficient for a twoports' active network [9]. Because of his discussion and state, the stability theory of generally two-port network became complete and rigorously. In 1992, Edwards and Sinsky brought up a single parameter to replace Rollet condition and the auxiliary conditions [10]. The single geometrically derived parameter is simplified the design work of RF circuits design. Güneş and Çetiner followed up his work in 1998, and presented a stability analysis for transistor with emphasis on the concept of having an unconditionally stable working area [11]. The rigorously derivation and theory of stability of general two-port active were discussed in these previous works. However, the $k$-factor that based on transistors' small-signal model (SSM) has not been discussed in these literatures. In the early 1975, Vendelin discussed the frequency of the peak $k$-factor in regard to GaAs MESFET using simple SSM [12], only a few of feedback parameters were used to represent the $k$-factor. In 1999, Gharpurey and Viswanathan had shown the formula of $k$-factor that is in terms of SSM parameters of BJT with common-emitter configuration [13]. Based on these formulas, tasks of circuit and device design are more easily, and gives more useful pictures to perform a stable transistor as evaluating the processes. However, the discussion on the stability in terms of the SSM parameters of CMOS is still deficient.

In this paper, the stability of MOSFET was discussed based on the criteria mentioned in literature [9]. In addition, the stability factor associated with the microwave SSM of MOSFET was derived, including the gate resistance and channel charging resistance. Since the circuit parameters such as $Y$-parameter and cutoff frequency can be used to further verify the validity of MOSFETs RF modeling [14,15], the stability factor derived in conjunction with SSM parameters is beneficial for verifying the accuracy of device modeling. Besides, for the first time, this study provides some new insights into the effects of biasing and scaling parameters on stability. Subsequently, the stability factor derived in terms of SSM parameters was verified by our experimental results. These new insights as well as the associated comprehension can be useful and valuable for device and circuit design.

\section{Experimental and measurement}

MOS transistors used in this study were fabricated on 6-in. wafers using $0.35 \mu \mathrm{m}$ logic technology. Gate oxide and polysilicon gate were $70 \AA$ and $0.25 \mu \mathrm{m}$ in thickness respectively. A sidewall spacer having a width of 0.21 $\mu \mathrm{m}$ was used with source/drain and gate treated by a salicide process using Ti/TiN material. The final gate's sheet resistance was $2-5 \Omega / \square$ after salicidation process.

The common-source configuration was generally used in a testing of RF device pattern. The gate and drain of the test transistor in this work are connected to the port- 1 and port- 2 respectively, while the source and substrate are connected to the ground pads. In addition, the pads are configured in ground-signal-ground for each port to perform $S$-parameter measurements. The $S$-parameter measurements are executed using an HP8510C network analyzer. Operationally, the range of sweep frequency is between 0.3 and $29.7 \mathrm{GHz}$, and the source power is $-10 \mathrm{dbm}$ with $20 \mathrm{db}$ attenuation to avoid non-linear distortion. The measurement system was first calibrated by short-open-load-through (SOLT) procedures on impedance-standard substrate (ISS); and testing devices were de-embedded by open and short dummy patterns [16-19].

\section{Results and discussion}

\subsection{Calculation of new stability-factor model}

The first step in designing RF active circuit is to determine the stability of transistor, this is especially important for amplifier design. Fig. 1 illustrates the twoport network to depict the stability of a transistor. The input and output reflection coefficients $\Gamma_{\text {IN }}$ and $\Gamma_{\text {OUT }}$ were given as follow [3],

$$
\begin{aligned}
& \Gamma_{\mathrm{IN}}=S_{11}+\frac{S_{12} S_{21} \Gamma_{\mathrm{L}}}{1-S_{22} \Gamma_{\mathrm{L}}} \\
& \Gamma_{\mathrm{OUT}}=S_{22}+\frac{S_{12}+S_{21} \Gamma_{\mathrm{S}}}{1-S_{11} \Gamma_{\mathrm{S}}}
\end{aligned}
$$




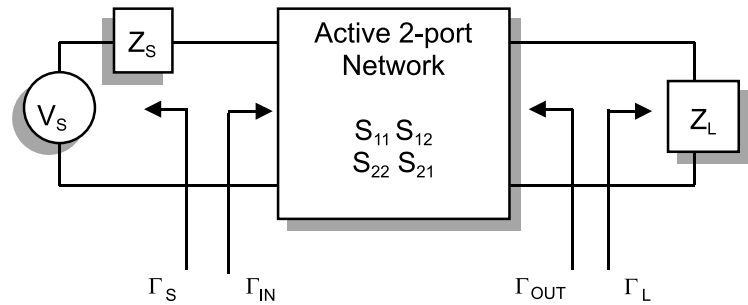

Fig. 1. A two-port active network illustrating the reflection coefficients.

where $S_{11}, S_{12}, S_{21}$ and $S_{22}$ are the scattering parameters of transistor, $\Gamma_{\mathrm{L}}$ and $\Gamma_{\mathrm{S}}$ are the load reflection and source reflection coefficient respectively, as denoted in Fig. 1. The network becomes unstable (or oscillates) if $\left|\Gamma_{\mathrm{IN}}\right|>1$ or $\left|\Gamma_{\mathrm{OUT}}\right|>1$ for possible passive load or source impedance. This implies the fact that the real part of input (or output) impedance is negative, and hence, somewhat can be imaged as the input (or output) impedance "generates energy" feedback to generator (or load). Hence, to obtain both $\left|\Gamma_{\mathrm{IN}}\right|<1$ and $\left|\Gamma_{\text {OUT }}\right|<1$, adding a positive resistive matching network can tuned the unstable network to be stable. However, the added resistive network increases the noise level of the network and lowering both the power gain and bandwidth of amplifier. Another technique is neutralizing which is proposed to cancel $S_{12}$ by adding feedback network. The reduced $S_{12}$ can then increase the $k$-factor. This usually performed by connecting an inductor between gate and drain to resonate gate-to-drain capacitance. This neutralizing technique will reduce the power gain but increase the bandwidth. The conditions required for this two-port network to be unconditionally stable are that both $\left|\Gamma_{\mathrm{IN}}\right|$ and $\left|\Gamma_{\mathrm{OUT}}\right|$ become less than unity for all possible $\Gamma_{\mathrm{L}}$ and $\Gamma_{\mathrm{S}}$ values with load and source impedance in a positive real part. Moreover, the accepted conditions necessary and sufficient for a two-port network to be unconditionally stable are [9-11]

$k=\left[\frac{1-\left|S_{11}\right|^{2}-\left|S_{22}\right|^{2}+|\Delta|^{2}}{2\left|S_{12} S_{21}\right|}\right]>1$

and

$|\Delta|=\left|S_{11} S_{22}-S_{12} S_{21}\right|<1$

where $k$ is the stability factor of the transistor and it is commonly used to gauge the stability of both devices and circuits in the area of computer aided design. The unconditionally stable criteria shown in Eqs. (3) and (4) are the most simplified forms to be widely used. However, a formulation containing admittance can be used to obtain more emphasized insights for stability factor, $k$, as shown in Eq. (5) $[20,21]$.

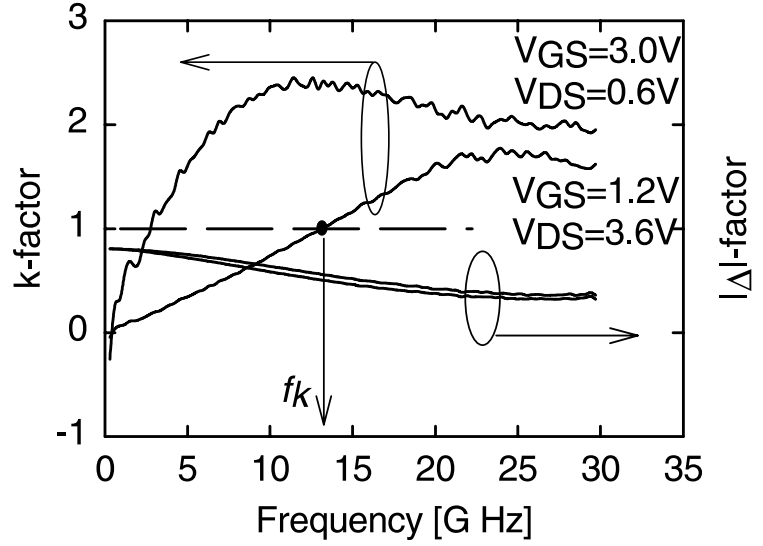

Fig. 2. Illustration of unity-stability-factor frequency. Two bias conditions were performed (i.e. $V_{\mathrm{gs}}=1.2 \mathrm{~V}, V_{\mathrm{ds}}=3.0 \mathrm{~V}$ and $\left.V_{\mathrm{gs}}=3.0 \mathrm{~V}, V_{\mathrm{ds}}=0.6 \mathrm{~V}\right)$. The range of swept frequency is $0.3-$ $29.7 \mathrm{GHz}$.

$k=\frac{\operatorname{Re}\left(Y_{11}\right) \operatorname{Re}\left(Y_{22}\right)-\operatorname{Re}\left(Y_{12} Y_{21}\right)}{\left|Y_{12} Y_{21}\right|}$

Fig. 2 shows the measurement data of $k$-factor and $|\Delta|$-factor versus the sweeping frequency ranging from 0.3 to $29.7 \mathrm{GHz}$ for a MOSFET at different biases. The $|\Delta|$-factor is normally less than 1 , which satisfies the unconditionally stable condition as shown in Eq. (4). Notice that $|\Delta|$-factor is less than one for each device observed. However, the $k$-factor decreases with decreasing frequency and becomes less than 1 in the lower frequency regime (i.e., no longer unconditionally stable). Hence, the $k$-factor will be the main focus in the further discussion of this paper.

The SSM of a MOSFET is shown in Fig. 3, and the $Y$-parameter of the SSM can be expressed by using the form found in [8]. Herein, only the intrinsic part of MOSFET is considered for calculating efficient. In addition, gate resistance, $R_{\mathrm{g}}$, and channel charging resistance, $r_{\mathrm{i}}$, were also included in our calculation to examine their effects on transistor's stability. Afterwards, the $Y$-parameter that was described in [8] is used to substitute in Eq. (5). With the second order approximation performed to the frequency, the $k$-factor in terms of SSM parameters can be calculated in the following:

$$
\begin{gathered}
k \cong \frac{\omega\left(2 R_{\mathrm{g} g} C_{\mathrm{ds}} C_{\mathrm{g}}^{2}+2 r_{\mathrm{i}} g_{\mathrm{ds}} C_{\mathrm{gs}}^{2}+2 R_{\mathrm{g}} g_{\mathrm{m}} C_{\mathrm{g}} C_{\mathrm{gd}}+C_{\mathrm{gd}}^{2}\right)}{\left[\omega^{2}\left(C_{\mathrm{gd}}^{2}+2 R_{\mathrm{g}} g_{\mathrm{m}} C_{\mathrm{g}} C_{\mathrm{gd}}\right)^{2}+g_{\mathrm{m}}^{2} C_{\mathrm{gd}}^{2}\right]^{1 / 2}} \\
=\frac{\left(2 R_{\mathrm{g}} g_{\mathrm{ds}} C_{\mathrm{g}}^{2}+2 r_{\mathrm{i}} g_{\mathrm{ds}} C_{\mathrm{gs}}^{2}+2 R_{\mathrm{g}} g_{\mathrm{m}} C_{\mathrm{g}} C_{\mathrm{gd}}+C_{\mathrm{gd}}^{2}\right)}{\left[\left(C_{\mathrm{gd}}^{2}+2 R_{\mathrm{g}} g_{\mathrm{m}} C_{\mathrm{g}} C_{\mathrm{gd}}\right)^{2}+\left(g_{\mathrm{m}} C_{\mathrm{gd}} / \omega\right)^{2}\right]^{1 / 2}}
\end{gathered}
$$




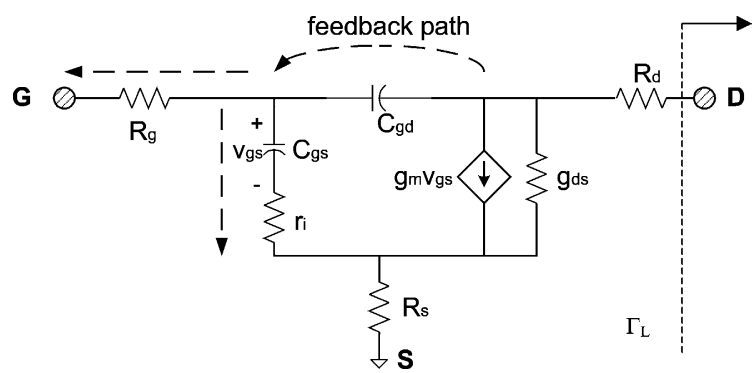

Fig. 3. The SSM of MOSFET for the derivation of $k$-factor. The feedback paths from the output toward the input port were also indicated.

where $g_{\mathrm{m}}$ represents the gate transconductance, $g_{\mathrm{ds}}$ is the output conductance, $C_{\mathrm{g}}$ is equal to $C_{\mathrm{gg}}+C_{\mathrm{gd}}$, and $\omega$ is the swept radius frequency. The derived $k$-factor ignores the effect of in-series source/drain resistance. In practical, the drain and source resistance will increase the value of $k$-factor and the device tends to stable. It can be seen from Eq. (6) that the $k$-factor is increased with increasing frequency, and the $k$-factor is dominated by both transconductance and gate-to-drain capacitance in lower frequency region. The channel charging resistance that represents the non-quasi-static effect of channel charges would increase the $k$-factor as observed in Eq. (6). Since $r_{\mathrm{i}} C_{\mathrm{gs}}$ is the charging time constant of channel charges, this means $k$-factor will be larger in longer channel or higher speed signals. Another of interest parameter in Eq. (6) is the gate resistance, and it was also found that increase of $R_{\mathrm{g}}$ could cause the $k$ factor to increase.

Next, we define the unity stability-factor frequency, $f_{k}$, of which it is the frequency with $k=1$ as indicated by the arrow sign in Fig. 2. By using the concept of unity stability-factor frequency, the insight of transistor's stability can be fulfilled more significantly and the boundary between unconditionally stable and potentially stable regions can be observed more clearly. Unity stabilityfactor frequency can be treated as the bandwidth of potentially unstable of transistor and also can be denoted as the corner frequency of MSG and MAG. For many of the applications, the bandwidth of potentially unstable is required as small as possible. The unity stability-factor frequency can be derived from Eq. (6) by setting $k=1$, as expressed in Eq. (7) with other terms included in the dominator of Eq. (7). This result arises from the fact that the capacitance of intrinsic gate to drain reduces drastically in saturation region. For convenience' sake, Eq. (7) can be simplified to more made brief expressions as shown in Eqs. (8a) and ( $8 \mathrm{~b})$ because the MOSFET are operated in saturation region for most $\mathrm{RF}$ active circuits,

$\left.f_{k} \approx g_{\mathrm{m}} d /\left\{2 \pi C_{\mathrm{g}} \sqrt{8 g_{\mathrm{ds}} R_{\mathrm{g}} d s\left[g_{\mathrm{m}} s^{2}\left(R_{\mathrm{g}}+r_{\mathrm{i}}\right)+d s\left(3 g_{\mathrm{m}} R_{\mathrm{g}}+1\right)\right.}\right]\right\}$

$$
\approx f_{t} d / \sqrt{\left[8 g_{\mathrm{ds}} R_{\mathrm{g}} d s\left(g_{\mathrm{m}} s^{2}\left(R_{\mathrm{g}}+r_{\mathrm{i}}\right)+d s\left(3 g_{\mathrm{m}} R_{\mathrm{g}}+1\right) s\right]\right.}
$$

where $f_{t}=g_{\mathrm{m}} /\left(2 \pi C_{\mathrm{g}}\right)$ is the cut-off frequency of MOSFET. The derived unity stability-factor frequency in Eqs. (8a) and (8b) is especially suitable for saturation regime since the corresponding $d^{3}$, and $d^{2}$ terms located in the dominator were ignored. From Eq. (8b), $f_{k}$ is proportional to $f_{t}$, indicating that the device falls off more from a wider and potentially unstable frequency region with larger bandwidth. The measurement data of $f_{k}$ versus $f_{t}$ at different $V_{\mathrm{gs}}$ and $V_{\mathrm{ds}}$ confirms this correlation as shown in Fig. 4. In Fig. 5, parameters $d$ and $s$ with which they can strongly affect the stability as expressed in Eqs. (8a) and (8b) are the feedback related parameters in SSM $[12,22,23]$. It can be seen in Fig. 5 that smaller $C_{\mathrm{gd}} / C_{\mathrm{g}}$ or larger $C_{\mathrm{gs}} / C_{\mathrm{g}}$ would result in smaller $f_{k}$ to be derived from Eq. (8a); therefore, smaller $d$ and larger $s$ are beneficial to stable RF amplifier design. In fact, capacitance $C_{\mathrm{gd}}$ provides a feedback path and the reflected energy was coupled from the drain toward the gate and vanished by $R_{\mathrm{g}}$ if stable as illustrated in Fig. 3. The other feedback energy will through the gate-to-source capacitance to the ground and vanished too.

\subsection{The effects of bias and scaling parameter on stability}

Since Si-based BJTs have been employed to building the RF front-end blocks in most RF applications, hence, compare its stability with CMOS can be valued to establish the instable issue of CMOS. The $k$-factor and $|\Delta|$-factor of BJT were measured by using different $V_{\text {be }}$ biases as shown in Fig. 6. The BJT under testing was

$f_{k}=\frac{g_{\mathrm{m}} d}{4 \pi C_{\mathrm{g}} \sqrt{g_{\mathrm{ds}} R_{\mathrm{g}}\left[\left(g_{\mathrm{ds}}+g_{\mathrm{ds}} \frac{r_{\mathrm{i}}}{R_{\mathrm{g}}}\right) s^{4}+2\left(3 g_{\mathrm{m}} R_{\mathrm{g}}+1\right) d^{3} s+2 g_{\mathrm{m}}\left(R_{\mathrm{g}}+r_{\mathrm{i}}\right) d s^{3}+\left(2 g_{\mathrm{m}} r_{\mathrm{i}}+6 g_{\mathrm{m}} R_{\mathrm{g}}+R_{\mathrm{g}}+\frac{r_{\mathrm{i}}}{R_{\mathrm{g}}}+1\right) d^{2} s^{2}\right]}}$

where $d=C_{\mathrm{gd}} / C_{\mathrm{g}}, s=C_{\mathrm{gs}} / C_{\mathrm{g}}$. Eq. (7) also ignored the contribution from $d^{4}$, since it is very small as compared fabricated with $0.8 \mu \mathrm{m}$ double polysilicon BiCMOS process and have an emitter area of $0.8 \times 10 \mu \mathrm{m}^{2}$. 


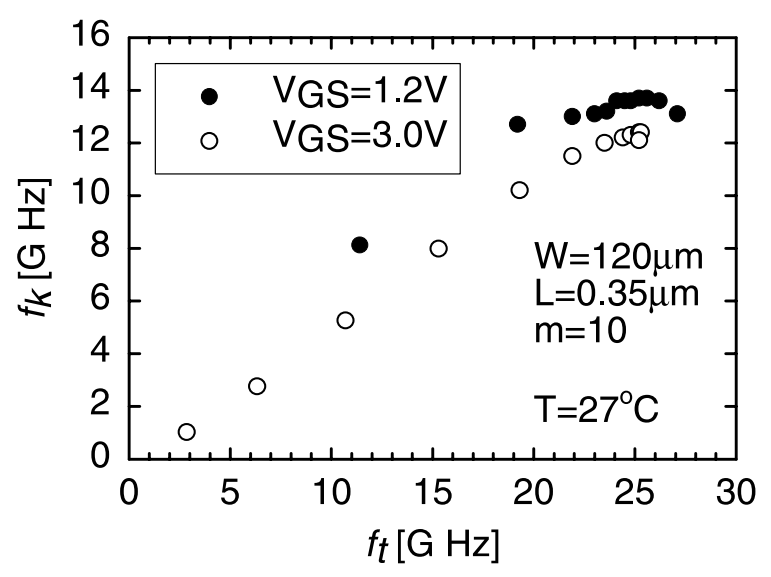

Fig. 4. Unity-stability-factor frequency versus unity currentgain frequency with various biases.

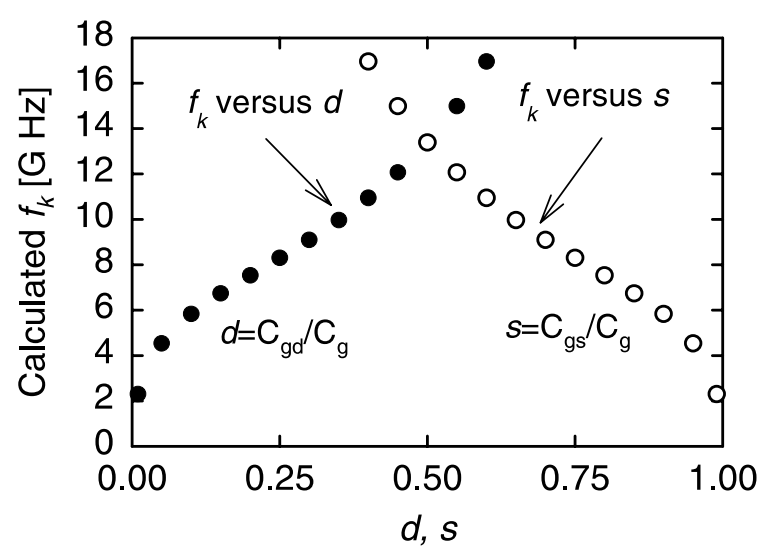

Fig. 5. Feedback parameters $d=C_{\mathrm{gd}} / C_{\mathrm{g}}$ and $s=C_{\mathrm{gs}} / C_{\mathrm{g}}$ versus the calculated $f_{k}$.

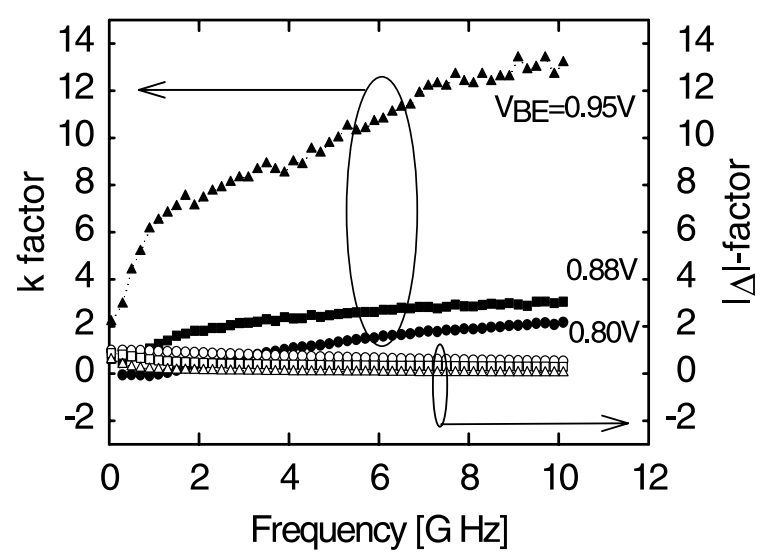

Fig. 6. $k$-factor and $|\Delta|$-factor versus swept frequency for npnBJT with emitter area $0.810 \mu^{2}$. The bias conditions are $V_{\text {ce }}=3.0 \mathrm{~V}$ and $V_{\text {be }}=0.80,0.88$ and $0.95 \mathrm{~V}$ respectively.
Similar to the MOSFET, $|\Delta|$-factor of BJTs were always less than unity as observed, and hence satisfies one of the stability criterions, that is Eq. (4). Similar to aforementioned stability of MOSFET, $k$-factor becomes the only one factor to determine the stability of transistor. It is found that $f_{k}$ equals to $4 \mathrm{GHz}$ at $V_{\text {be }}=0.8 \mathrm{~V}$ and $1 \mathrm{GHz}$ at $V_{\text {be }}=0.88 \mathrm{~V}$. The broadband ( $f_{k}$ is less than $1 \mathrm{GHz}$ ) to be unconditionally stable is achieved with $V_{\text {be }}=0.95 \mathrm{~V}$. This is beneficial for RF amplifier design without lossy matching network. However, broadband to be unconditionally stable in MOSFET cannot be obtained under any biases. Basically, MOSFET is inherently potentially unstable at low and intermediate frequency regions as mentioned previously. Fig. 7 shows the mapping $\Gamma_{\mathrm{IN}}$ contour in the $\Gamma_{\mathrm{L}}$ plane of specific interesting frequencies (or called output stability circle). The bias conditions are $V_{\mathrm{gs}}=3 \mathrm{~V}$ and $V_{\mathrm{ds}}=3.3 \mathrm{~V}$. While at $1.8 \mathrm{GHz}$, the unstable region is almost half of the Smith chart and is associated with inductive reactance. This implies the fact that the allowed matching network is restricted to design stable amplifiers. With increased frequency, the unstable area decreased and becomes unconditionally stable up to $14.4 \mathrm{GHz}$. To perform a stable amplifier design, a safe way is to add resistive matching network. For instance, one can add in-series $50 \times 5=250 \Omega$ resistor to the output port of two-port network to achieve unconditionally stable. The added $250 \Omega$ resistor can further ensure that two-port network is unconditionally stable at 2.4 and $5.7 \mathrm{GHz}$ and so on. However, resistive matching to ensure broadband unconditional stable is

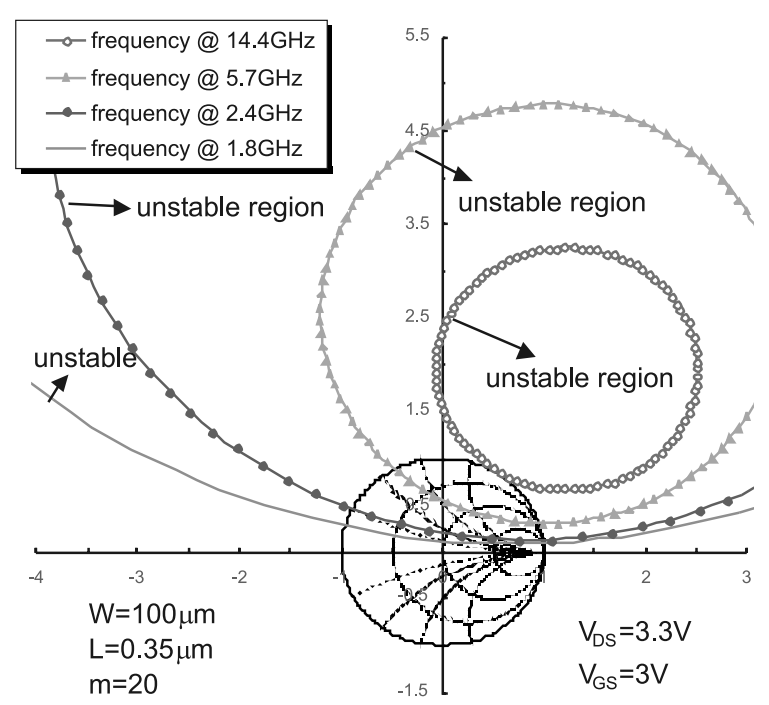

Fig. 7. Output stability circle of MOSFET with $W=100 \mu \mathrm{m}$, $L=0.35 \mu \mathrm{m}$ and $m=20$. The regions inside of circles represent the unstable region for each specific frequency. The Smith chart is the unity $\Gamma_{\mathrm{L}}$ circle. 
not an efficient method because of the increased thermal noise and reduced power gain. Another approach is to design the amplifier to be conditionally stable. This approach needs lots of trial and error iterations, and needs precise passive components in ICs and accurate RF models. However, precise components and accurate model are the two major bottlenecks in IC level. Hence, in our view of points, optimization on unconditionally stable related to transistors' biases and geometry might be the more efficiency approach.

In Figs. 8 and $9, f_{k}$ is found to increase with increasing $V_{\mathrm{ds}}$, and tends to saturate at higher $V_{\mathrm{ds}}$. Recalled Eq. (8a) that can be helpful to analyze the bias dependence. Since $R_{\mathrm{g}}$ and $C_{\mathrm{g}}$ were almost constant with $V_{\mathrm{ds}}$, the effect of bias is dominated by $g_{\mathrm{ds}}$ and $g_{\mathrm{m}}$. Observed the denominator and numerator of Eq. (8a) respectively, $g_{\mathrm{ds}}$ decreased with increased $V_{\mathrm{ds}}$ and $g_{\mathrm{m}}$ increased with $V_{\mathrm{ds}}$, the denominator has weaker bias dependence compared to numerator. Hence, the increased $f_{k}$ is mainly due to increased $g_{\mathrm{m}}$. In fact, based on Eq. (8a), the unity-

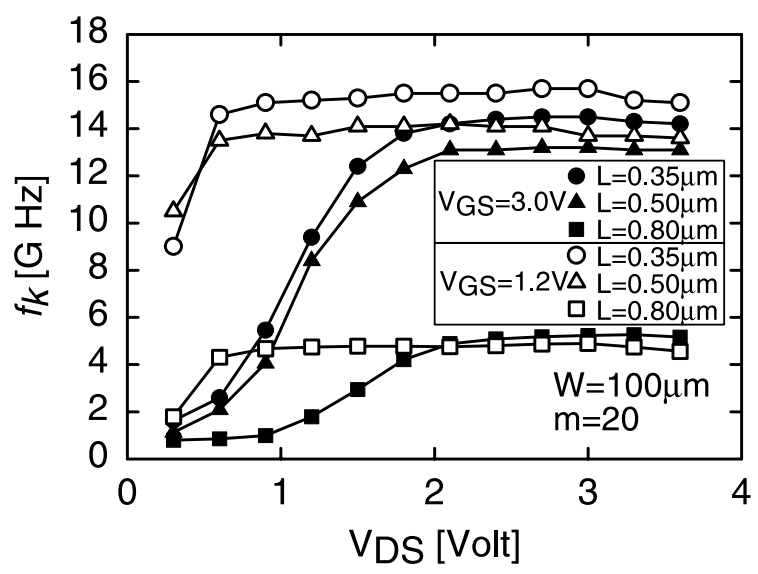

Fig. 8. $f_{k}$ versus $V_{\mathrm{ds}}$ for different biases and gate lengths.

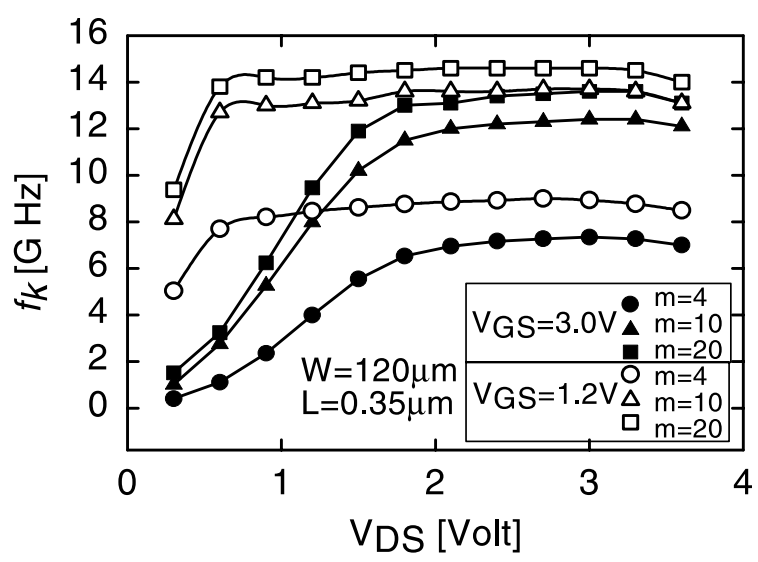

Fig. 9. $f_{k}$ versus $V_{\mathrm{ds}}$ for different biases and finger numbers. stability-factor frequency is roughly proportional to $\left(g_{\mathrm{m}} / g_{\mathrm{ds}}\right)^{1 / 2}$, which is similar to the low-frequency voltage gain from common-source amplifier, i.e., $\left(g_{\mathrm{m}} / g_{\mathrm{ds}}\right)$. For the straightforward sense, transistors with higher power gain should have larger MSG and MAG, and hence, higher $f_{k}$. Hence, stability should be the main focus when designing the RF amplifier with common-source configuration. At higher $V_{\mathrm{ds}}$, the tail of $f_{k}$ is reduced because of the slight increase of $g_{\mathrm{ds}}$, caused by the impact ionization [24]. In addition, similar analysis can be used to observe $V_{\mathrm{gs}}$ dependence of $f_{k}$. Again, observed from Fig. 8 and $9, f_{k}$ at $V_{\mathrm{gs}}=1.2 \mathrm{~V}$ is higher than that at $V_{\mathrm{gs}}=3.0 \mathrm{~V}$ because a maximum $g_{\mathrm{m}}$ is obtained at $V_{\mathrm{gs}}=$ $1.2 \mathrm{~V}$.

The significance of the layout geometry on the RF FOM's has been discussed evident in Refs. [4-6,8,18,25], and included their optimization. RF performance such as $F_{\mathrm{t}}, F_{\max }$ and $\mathrm{NF}_{\min }$ can be obviously improved with shrinking technologies, and hence, makes CMOS technology become attractive in RF applications. The intrinsic cut-off frequency is proportional to $1 / L^{2}$ without velocity saturation and $1 / L$ with velocity saturation [26]. In addition, $F_{\max }$ is proportional to $1 / \sqrt{L}$ roughly [8]. However, as mentioned previously, stability will be a trade-off parameter as implement a RF circuit especially the amplifier. Fig. 8 shows the length dependence of $f_{k}$ with fixed finger number $(m)$ and total gate width. The unity-stability-factor frequency was increased with reduced gate length. The stability issue will be emerged for short channel devices and might make the RF amplifier design difficult. Fig. 10 shows the comparison of MSG and MAG of $0.18 \mu \mathrm{m}$ and $0.13 \mu \mathrm{m}$ technologies of CMOS with the same total gate width and finger number. The broader bandwidth of MSG of $0.13 \mu \mathrm{m}$ technology implies broader potentially unstable bandwidth,

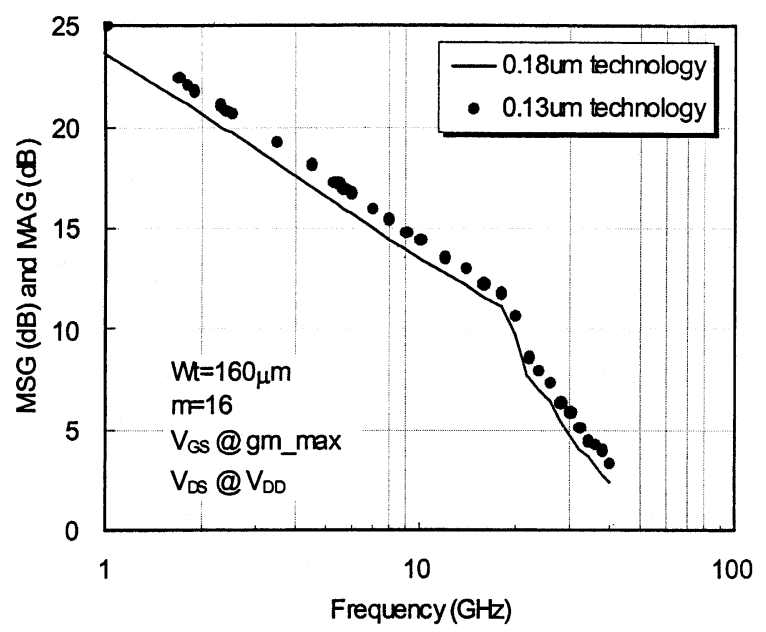

Fig. 10. MSG and MAG of 0.18 and $0.13 \mu \mathrm{m}$ technologies of MOSFET. 


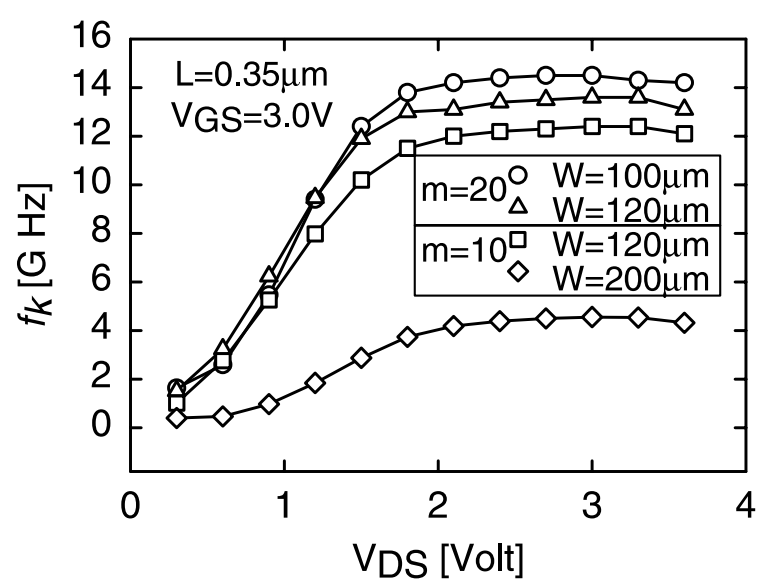

Fig. 11. Gate width effects on $f_{k}$ with respect to different finger numbers.

and hence, larger unstable region on the Smith chart. Even though, selecting proper devices' dimension, that is, finger number and total gate width, can perform optimization on stability. Smaller finger number (e.g., $m=4$ in Fig. 9) can reduce $f_{k}$ down to $6 \mathrm{GHz}$ with $V_{\mathrm{ds}}=V_{\mathrm{gs}}=3 \mathrm{~V}$. Widen total gate width $(W=200 \mu \mathrm{m}$ in Fig. 11) also can reduce $f_{k}$ down to $4 \mathrm{GHz}$ with $V_{\mathrm{ds}}=V_{\mathrm{gs}}=3 \mathrm{~V}$. However, these rule somewhat tends to increased the gate resistance and degrade the $\mathrm{NF}_{\text {min }}$ and $F_{\max }$. One might recall Eq. (8a), since $R_{\mathrm{g}}$ is reverse proportional to $f_{k}$, our measurement data coincides the derived model. In practical, even the increased finger number can increase the $F_{\max }$, but MSG cannot be improved. As shown in Ref. [25], each finger number keeps the same MSG until the MAG appear and increased $F_{\max }$ with increased finger number. Herein, we suggest that technology in progressing $R_{\mathrm{g}}$, such as T-shape gate and metal gate, can be used to reduce the $\mathrm{NF}_{\min }$, and the finger number and gate width can be used to optimizing stability. In addition, to the extent that both $g_{\mathrm{m}}$ and $g_{\mathrm{ds}}$ are proportional to $W / L$ plus $R_{\mathrm{g}}$ is proportional to $W /\left(L m^{2}\right), f_{k}$ increases with decreasing $W$ and $L$ and becomes larger with increasing $m$. Experimental measurements of $f_{k}$ confirm that a wider width and a smaller finger number are desirable for achieving a broader and unconditionally stable band.

\section{Conclusion}

A comprehensive study on MOS device stability for RF application has been performed for the first time. The simplified calculation of $k$-factor based on the SSM including gate resistance was achieved. Meanwhile, unity stability-factor frequency was also proposed to gain an insight into the stability of MOSFET. Furthermore, the effects of both biasing and scaling parameters on stability were also characterized. The results indicate that devices with short and narrow channel and large finger number can increase their unity stability-factor frequency; hence, these devices can make the design of $\mathrm{RF}$ circuits more complicated. It is proposed from the results of this study that a RF active circuit operating at $2.4 \mathrm{GHz}$ and above can be unconditionally stable by adopting proper scaling and biasing conditions without the need of an additional stability-related matching network.

\section{Acknowledgements}

This research is supported by National Science Council, Taiwan, ROC, and under contract NSC902215-E009-112.

\section{References}

[1] Chin D. Executing system on a chip: requirements for a successful SOC implementation. Proc IEDM 1998:3.

[2] Razavi B. CMOS technology characterization for analog and RF design. IEEE J Solid-State Circ 1999;34(3):268.

[3] Vendelin GD, Pavio AM, Rohde UL. Microwave circuit design using linear and nonlinear techniques. New York: Wiley; 1990.

[4] Voinigescu SP, Tarasewicz SW, MacElwee T, Ilowski J. An assessment of the state-of-the-art $0.5 \mu \mathrm{m}$ bulk CMOS technology for RF applications. Proc IEDM 1995:721.

[5] Saito M, Ono M, Fujimoto R, Tanimoto $\mathrm{H}$, Ito $\mathrm{N}$, Yoshitomi $\mathrm{T}$, et al. $0.15-\mu \mathrm{m}$ RF CMOS technology compatible with logic CMOS for low-voltage operation. IEEE Trans Electron Dev 1998;45(3):737-42.

[6] Morifuji E, Momose HS, Ohguro T, Yoshitomi T, Kimijima H, Matsuoka F, et al. Future perspective and scaling down roadmap for RF CMOS. Symp VLSI Tech Dig 1999:163.

[7] Chatterjee A, Vasanth K, Grider DT, Nandakumar M, Pollack G, Aggarwal R, et al. Transistor design issues in integrating analog functions with performance digital CMOS. Symp VLSI Tech Dig 1999:147.

[8] Manku T. Microwave CMOS-Device physics and design. IEEE J Solid-State Circ 1999;34(3):277.

[9] Woods D. Reappraisal of the unconditional stability criteria for active 2-port networks in terms of $S$ parameters. IEEE Trans Circ Syst 1976;23(2):73.

[10] Edwards ML, Sinsky JH. A new criterion for linear 2port stability using a single geometrically derived parameter. IEEE Trans Microwave Theory Tech 1992;40(12): 2303-11.

[11] Günes F, Çetiner BA. Smith chart formulation of performance characterization for a microwave transistor. IEE Proc-Circ Dev Syst 1998;145(6):419-28.

[12] Vendelin GD. Circuit model for the GaAs MESFET valid to $12 \mathrm{GHz}$. Electron Lett 1975;11(3):60.

[13] Gharpurey R, Viswanathan TR. Design of front-end RF circuits. Southwest Symp on Mixed-Signal Design. 1999. p. 134-9. 
[14] Ou JJ, Jin X, Ma I, Hu C, Gray PR. CMOS RF modeling for $\mathrm{GHz}$ communication IC's. Symp VLSI Tech Dig 1998:94.

[15] Cheng Y, Schroter M, Enz C, Matloubian M, Pehlke D. RF modeling issues of deep-submicron MOSFETs for circuit design. Proc 5th International Conference on SolidState and Integrated Circuit Technology. 1998. p. 416.

[16] Cariton DE, Gleason KR, Strid EW. Microwave wafer probing. Microwave J 1985.

[17] Jones K, Strid EW. Verify wafer probe reference planes for MMIC testing. Microwaves and RF 1988.

[18] Biber C, Morf T, Benedickter H, Lott U, Bächtold W. Microwave frequency measurements and modeling of MOSFETs on low resistivity silicon substrates. Proc IEEE ICMTS 1996;9:211.

[19] Kolding TE. On-wafer calibration techniques for gigahertz CMOS measurements. Proc IEEE ICMTS 1999; 12:105.
[20] Rollett JM. Stability and power-gain invariants of linear twoports. IRE Trans Circ Theory 1962;CT-9:29.

[21] $\mathrm{Ku} \mathrm{WH}$. Unilateral gain and stability criterion of active two-ports in terms of scattering parameters. Proc IEEE 1966;54:1617.

[22] Jin X, Ou J-J, Chen C-H, Liu W, Deen MJ, Gray PR, $\mathrm{Hu}$ C. An effective gate resistance model for CMOS RF and noise modeling. Proc IEDM 1998:961.

[23] Johnson H. A high-frequency representation of the MOS transistor. Proc IEEE 1966;54:1970.

[24] Arora N. MOSFET models for VLSI circuit simulation. Wien, New York: Springer; 1993.

[25] Burghartz JN, Hargrove M, Webster CS, Groves RA, Keene M, Jenkins KA, et al. RF potential of a $18-\mu \mathrm{m}$ CMOS logic device technology. IEEE Trans Electron Dev 2000;47(4)

[26] Tsividis Y. Operation and modeling of the MOS transistor. 2nd ed. Singapore: McGraw-Hill; 1999. 